\title{
La Luçerna Yndyca de E. Sancho de Melgar y Santa Cruz. Manuscrito de finales del siglo XVII
}

La Luçerna Yndyca de E. Sancho de Melgar y Santa Cruz. Manuscrit de la fin du XVIIème siècle

The Luçerna Yndyca of E. Sancho de Melgar y Santa Cruz. Manuscript composed towards the end of the 17th century

\section{Gerald Taylor}

\section{OpenEdition}

\section{Journals}

Edición electrónica

URL: http://journals.openedition.org/bifea/2023

DOI: $10.4000 /$ bifea.2023

ISSN: 2076-5827

\section{Editor}

Institut Français d'Études Andines

\section{Edición impresa}

Fecha de publicación: 1 agosto 2010

Paginación: 413-425

ISSN: 0303-7495

\section{Referencia electrónica}

Gerald Taylor, «La Luçerna Yndyca de E. Sancho de Melgar y Santa Cruz. Manuscrito de finales del siglo XVII », Bulletin de l'Institut français d'études andines [En línea], 39 (2) | 2010, Publicado el 01 febrero 2011, consultado el 07 noviembre 2020. URL : http://journals.openedition.org/bifea/2023 ; DOI https://doi.org/10.4000/bifea.2023

\section{(c) $(1) \odot$}

Les contenus du Bulletin de l'Institut français d'études andines sont mis à disposition selon les termes de la licence Creative Commons Attribution - Pas d'Utilisation Commerciale - Pas de Modification 4.0 International. 


\title{
La Luçerna Yndyca de E. Sancho de Melgar y Santa Cruz. Manuscrito de finales del siglo XVII*
}

\author{
Gerald Taylor**
}

\begin{abstract}
Resumen
Se conoce a Sancho de Melgar por su Arte de la lengua general del Ynga llamada Qquechhua, publicado en Lima en 1691. Existe en la Biblioteca Nacional de Bogotá un manuscrito inédito del mismo autor llamado Luçerna Yndyca que contiene un estudio sobre la ortografía de la lengua general, un copioso léxico castellano-quechua (que es en realidad una sistematización del vocabulario de González Holguín) y traducciones del evangelio. Su interés principal, sin embargo, es el de ser testimonio de cómo un catedrático sanmarquino de esa época enseñaba la lengua general; se trata de una obra redactada en una mezcla barroca de latín y castellano, llena de digresiones eruditas.
\end{abstract}

Palabras clave: quechua, lengua general, filología, evangelización

\section{La Luçerna Yndyca de E. Sancho de Melgar y Santa Cruz. Manuscrit de la fin du XVIIème siècle}

\section{Résumé}

Sancho de Melgar nous est connu grâce à son Arte de la lengua general del Ynga llamada Qquechhua, publié à Lima en 1691. À la Bibliothèque Nationale de Bogotá se trouve un manuscrit inédit du même auteur: La Luçerna Yndyca, qui contient une étude sur l'orthographe de la lengua general, un lexique

Este artículo, redactado en el año 2007, se basa en una ponencia preparada para el Simposio «Les littératures didactique et dramatique en langues autochtones dans le Mexique et le Pérou coloniaux», organizado por el CELIA en colaboración con el Groupe de Recherche sur l'Amérique Latine y el Centre d'Anthropologie de la Universidad de Toulouse-Le Mirail y dirigido por Xavier Pello, el 9 y 10 de junio de 2006, en Toulouse-Le Mirail.

** CELIA (CNRS), IFEA. E-mail: gcptaylor@yahoo.fr 
volumineux castellan-quechua (qui est, en réalité, une systématisation du vocabulaire de González Holguín) et des traductions des Évangiles. Cependant, son principal intérêt est de se renseigner sur la façon dont, à cette époque, un professeur de l'université de San Marcos enseignait la lengua general : il s'agit d'une oeuvre rédigée dans un mélange baroque de latin et d'espagnol, remplie de digressions érudites.

Mots clés : quechua, lengua general, philologie, évangélisation

\title{
The Luçerna Yndyca of E. Sancho de Melgar y Santa Cruz. Manuscript composed towards the end of the 17 th century
}

\begin{abstract}
Sancho de Melgar is known to us thanks to his Arte de la Lengua General del Ynga Llamada Qquechhua, published in Lima in 1691. In the National Library of Bogota is to be found an unpublished manuscript of the same author, La Luçerna Yndyca, which contains a study of the spelling of the Lengua General, a copious Castellano-Quechua lexicon (in fact, a systematization of González Holguín's dictionary) as well as translations of the Gospels. However its principal interest is that of being a testimony to how a 17th century professor of the University of San Marcos taught the Lengua General. The manuscript is written in a baroque mixture of Latin and Spanish and is full of erudite digressions.
\end{abstract}

Key words: quechua, lengua general, philology, evangelisation

Gracias a una referencia contenida en la bibliografía de las lenguas aymara y quechua de Paul Rivet y Georges de Créqui-Montfort (Rivet \& Créqui-Montfort, 1951: 130-1), se sabe de la existencia de un importante manuscrito relacionado con la evangelización en quechua y conservado en Bogotá. Se trata de la Luçerna Yndyca del doctor Estéban Sancho de Melgar y Santa Cruz1, compuesta de unos 247 folios (casi 500 páginas de texto) y que contiene una selección de pasajes del Evangelio traducidos del latín al quechua. Según Rivet y Créqui-Montfort, en esa época se encontraba en el Archivo Nacional de Bogotá. Esta indicación dificultó durante muchos años su estudio, puesto que en los años 1990 las personas consultadas en el Archivo Nacional desconocían este documente que, finalmente, pudo ser localizado con a la ayuda del profesor Tulio Rojas en la Biblioteca Nacional de Colombia, Bogotá, donde había sido trasladado. Por intermedio

1 El título exacto, tal como aparece en el primer folio del manuscrito, es: LVÇERNA YNDYCA // Y Traducçion paraphrastica de / todos los Evangelios, que canta la Yglesia / segun el sentir de Santos Padres, Sagrados / Expossitores y Versiones Sacras.// Va al fin el Arte del mismo Author locu-/pletado, copiosso y curiosso Vocabulario. Ora-/ciones, y Cathecismos, Confessionario, / y Ritual// Por el Doctor D(o)n Estevan Sancho de Melgar y / Santa Cruz, Natural de esta Ciudad de los Reyes, / Capellan mas antiguo del Hospital Real de Santa Anna, / Doctor en Sagrada Theologia en la real Universidad de San / Marcos de dicha Ciudad, y Cathedratico en ella de la / lengua General de las Yndias de este Reyno, Cathedratico / tambien de dicha lengua en la Santa Yglesia Metropoli-/tana de dicha ciudad, y Examinador Synodal / de su Arzobispado.// Consagrasse, y dedica al Yll(ustrissi) mo S(eño)r, el Señor / D(octo)r D(o)n Antonio de Solonga, del Consejo de / su Magestad, Dignissimo Arzobispo de esta Me-/tropoli. 
de la investigadora Duna Troiani fue entonces posible conseguir una fotocopia, desafortunadamente de mala calidad, que reflejaba el estado deteriorado del original. La breve descripción que se presenta aquí, con la transcripción que la acompaña, se basa en esta fotocopia. La referencia bibliográfica actual comunicada por el señor Jaime Quevedo es el Ms 346 de la BNC (Biblioteca Nacional de Colombia).

La descripción que aparece en Rivet y Créqui-Montfort de la composición del manuscrito es bastante detallada y aquí añadiré sólo unos comentarios sobre su contenido. Se conoce al autor, Sancho de Melgar, por otra obra publicada en Lima en 1691: Arte de la lengua general del Ynga llamada Qquechhua. Se trata tal vez de la última parte de este manuscrito, a la que se refiere el autor en su título: «Va al fin el Arte del mismo Author», que efectivamente no se encuentra en el manuscrito que conocemos hoy. Una versión muy reducida de las reglas ortográficas del manuscrito que reproduzco aquí como documento anexo (anexo 1) se encuentra en el «Prólogo al Lector» de la publicación de 1691. En la dedicatoria al arzobispo don Antonio de Soloaga (f2r-ffrr), el autor expresa su deseo que el manuscrito sea publicado. Tal vez las autoridades limeñas no hayan sido convencidas por la utilidad de la publicación de una obra tan compleja (la gran época de las publicaciones de los tratados de evangelización en quechua - los años 40 del siglo XVII - ya era cosa del pasado) y el arte de 1691 fue todo lo que salió a luz del trabajo monumental de Sancho de Melgar.

Sabemos del autor que era, según indica la página del título del manuscrito, «Capellan mas antiguo del Hospital Real de Santa Anna, / Doctor en Sagrada Theologia en la real Universidad de San / Marcos de dicha Ciudad, y Cathedratico en ella de la / lengua General de las Yndias de este Reyno, Cathedratico / tambien de dicha lengua en la Santa Yglesia Metropoli-/tana de dicha ciudad, y Examinador Synodal / de su Arzobispado». Sabemos igualmente que el quechua no era su lengua materna («por no ser nativo en el idioma») y que la tarea que se había impuesto, emulando al Bautista como «luçerna» de los gentiles, implicó «dilatado tiempo al costo de prolija especulacion y cuidadosos desuelos»2.

El manuscrito tal como ha sido conservado en la BNC se compone de las partes siguientes:

- Título con referencias del autor y dedicatoria (f1r)3.

- Dedicatoria y justificación del trabajo (f2r- f6v).

- Prologo a los s(eño)res Curas (f7r-f10v).

- Bocabulario copioso y vtilissimo, assi para la facilidad en predicar, como / para la destreza en oyr a los penitentes en la confession (f11r-f42v).

- Indice de los Euangelios que contiene esta obra: el primero nu-/mero es el orden de cada Euang(eli)o el segundo es la pagina. (f43r-f44r)

2 Prologo a los s(eño)res Curas, $\mathrm{f} 7 \mathrm{r}$.

3 Las cifras impresas que indican los folios son modernas (comenzando por 00001). Solo la interpretación de los evangelios tiene una foliación antigua en la que f1r corresponde al f51 $\mathrm{r}$ de la numeración moderna y f198r al f247v moderno. 
- Reglas de la ortographia Indica, para el idiomista que aprende / por preceptos escriua con perfeccion y pronuncie con alguna pro-/priedad (f44v-f45v) (Su transcripción aparece como anexo de este artículo).

- Exposicion parafrastica de todos los Euangelios que canta la Iglesia confor-/me al sentir de S(antos)s Padres y sagrados Expositores, en la qual con natiuos parafra-/ sis y genuinos preceptos de este idioma, se da luz al cura de almas, para que con destre-/za y acierto pueda predicar y enseñar a sus feligreses las verdades catholicas que en los / santos Euangelios se contienen. (f46r-f50r) (Se compone de un Preludio de dos renglones y la exposición de una serie de nueve reglas para poder utilizar eficazmente la obra. La «Regla nona y Vltima» se refiere a la gramática del quechua y contiene una descripción detallada de la manera en que el Chinchaysuyo se distingue de la «lengua del Inga»).

A partir del f51r (o f1r de la foliación antigua) empieza la exposición en latín con su traducción al quechua de pasajes escogidos de los Evangelios considerados apropiados para fechas determinadas del año litúrgico. Solo el capítulo 26 de la pasión según San Mateo («Passio D(omi)ni nostri lesu Christi secundum Matthaeum. Cap. 26.»), f236r (f184)-f242v (f196v) se presenta en versión completa (la transcripción de un extracto de Mateo 22 [Evang. 108. Enseña lesus qual es el principal mandamiento de la ley: / confunde a los Phariseos con vna pregunta] ha sido agregada como documento anexo 2 a este artículo).

\section{EL AUTOR}

La hoja que contiene el título del manuscrito hace referencia al itinerario profesional del autor: «Natural de esta Çiudad de Los Reyes / Capellan mas antiguo del hospital real de Santa Anna, / Doctor en Sagrada Theologia en la Real Vniversidad de San / Marcos de dicha Çiudad, y Cathedratico en ella de la / lengua General de las Yndias de este Reyno, Cathedratico / también de dicha lengua en la Santa Iglesia Metropoli-/tana de dicha ciudad y Examinador Synodal / de su Arzobispado». No debe sorprender que la obra de un profesor con tanta experiencia sea una suma de erudición, esencialmente pedagógica y universitaria en su concepción. Es posible que el material de base de sus notas gramaticales y de su traducción y comentario de los Evangelios haya sido precisamente los apuntes para los cursos que dictaba en dichos centros de estudio. La abundancia de citas y referencias a los grandes exegetas bíblicos conocidos en aquella época muestra su gran erudición y también su formación universitaria. Desafortunadamente sabemos muy poco sobre el ambiente en los grandes centros de estudio donde un conocimiento de la lengua general era fundamental y que producían «curas de Indios», criollos como Sancho de Melgar y tal vez peninsulares también, capaces de componer sonetos en quechua, dedicados a sus colegas, de muy buena calidad lingüística a pesar de su forma y estilo hispánicos. No sabemos cuáles hayan sido sus lecturas. Solo podemos suponer a partir del análisis del manuscrito de Sancho de Melgar que su nivel de erudición era alto. El autor no era quechuahablante 
nativo. Aprendió el idioma probablemente en medios académicos semejantes a los donde enseñaba. No hay huella en su trabajo de la influencia de un quechua informal, hablado. Es entonces probable que la enseñanza de la lengua general en el contexto eclesiástico limeño fuera semejante a la del griego y del latín. El comentario del empleo respectivo de las partículas gramaticales, que forma parte de las reglas expuestas como introducción a la exposición de los Evangelios, implica un análisis profundo del sistema del idioma. Comentarios semejantes se encuentran en la justificación de las traducciones (con las variantes alternativas) que propone. Nunca dan la impresión de espontaneidad. Al contrario, todo está calculado a partir del análisis filológico del texto latino y los términos quechuas que busca son los que le parecen mejor corresponder a los matices del latín. Consecuentemente, el resultado parece ser a veces poco natural. En el siglo XVII, la lengua general — bajo la forma de diversas variantes_ era todavía un idioma hablado, el único que en la parte más importante de la colonia pudiera permitir la comprensión entre las diferentes categorías sociales, aunque probablemente en Lima, y hasta en el cercado, el castellano ya predominaba. Sin duda la lengua general, lengua franca de las clases populares y hablada en las comunidades al lado de los dialectos locales que también habían sufrido su influencia, difería del idioma erudito cuzqueñizante propuesto por los académicos de San Marcos. Se puede comparar el lenguaje de un documento como el manuscrito quechua de Huarochirí o los diversos textos quechuas de origen laico que se ha empezado a publicar en los últimos años con los sermonarios de Ávila y de Avendaño o las traducciones de los Evangelios que proporciona Sancho de Melgar.

\section{LOS CRITERIOS ORTOGRÁFICOS}

En la formulación de las reglas de la ortografía predominan los criterios pedagógicos de Sancho de Melgar. Difiere de las descripciones anteriores de la lengua por la indicación precisa de la estructura silábica del idioma, distinta de la del castellano. Por eso se sirve sobre todo del guión para mostrar que $r, \|$ e $y$, después de otra consonante, inician una nueva sílaba, puesto que el quechua no admite una secuencia de dos consonantes en la misma sílaba. Así, escribe chac-ra y choc-llo. En el caso de $y$, su criterio es un poco más complejo, ya que reconoce que para un hispanohablante una palabra como /tapya/ suena como una esdrújula y podría ser interpretada fonéticamente como ['tapiya], así pone el guión a ambos lados de y y escribe tap-y-a. De la misma manera, separa las dos $c$, que transcriben respectivamente /q/y/s/ en rec-cini/riqsini/ «conozco» por un guión.

Consciente de la necesidad de conservar los valores cuzqueños de la «lengua del Inga», emplea, aunque de manera un poco modificada, la grafía consagrada por los grandes quechuistas de los años 40 del siglo XVII (Ávila, Avendaño y Jurado Palomino) y distingue las oclusivas aspiradas y las glotalizadas. Sin embargo, solo en el caso de la bilabial su sistema funciona completamente: pacha «tiempo» y ppacha /p’acha/ «vestido». Expresa la aspirada mediante el guión seguido por $h$ : 
p-hucuni /phukuni/ «soplo»4. tt y cc/qqu representan respectivamente /th, $\mathrm{t}^{\prime} /$ y /q, qh, q'/ así como /kh, k'/. El recurso a los alófonos de /i/ y de /u/, es decir de $<\mathrm{e}>$ y de $\langle\mathrm{o}>$, permite distinguir entre la velar y la uvular en palabras como qquipu /khipu/ «nudo» y qquepa /qhipa/ «último», pero, cuando éstas preceden a /a/, solo la familiaridad con el lexema muestra que la secuencia inicial de ccari «varón» representa /qh/ y no /kh/, /k'/, /q/ o /q'/. Algunas grafías del vocabulario son insólitas y así encontramos qu-huyapayacc para /khuyapayaq/ «piadoso». La grafía chh representa /ch'/: chhussacc /ch'uŝaq/ «vacío». No hay entrada alguna en el vocabulario que corresponda a una palabra pronunciada hoy en los dialectos sureños con una africada aspirada /chh/.

Una innovación interesante del sistema gráfico empleado por Sancho de Melgar es la representación de la semivocal bilabial / w/ por vv. El ambiente cosmopolita del mundo de la evangelización de aquella época explica esta opción, a la que llegó «confi-/riendo este punto con vn sujeto muy docto, de singular ingenio, gran erudita, insigne no so-/lo en los idiomas Latino, Germanico, Frances, y otros desta Europa, sino tambien consuma-/do en los dos generales de este reyno qquechhua y Aymara, me asegurô que la pronun-/ciacion del Indio con estas sylabas era la misma que el vva, vve; vvi de los estrangero» (véase anexo 1).

El rechazo de $k$, empleada por González Holguín, para notar la oclusiva uvular /q/ corresponde a una reacción semejante a la expresada por Francisco de Ávila en la «Prefación» a su sermonario. No toma en consideración la posible utilidad del empleo de este signo en vez de la confusión evidente creada por el conjunto cc/qqu, sino basa su crítica en la pronunciación contemporánea de las voces Kalendae y Kyrie. Es verdad que González Holguín no es muy rigoroso en sus criterios ortográficos y encontramos «mar, laguna» traducida por las dos grafías ccocha y kocha. En la última década del siglo XIX, Middendorf resucita el signo $k$ para representar la uvular, manteniendo c y qu para la velar (Middendorf, 1970 [1890]).

\section{EL VOCABULARIO}

El manuscrito de Sancho de Melgar contiene un vocabulario importante castellanolengua general, 31 folios, o sea 62 páginas a doble columna. La base del trabajo del autor es el Vocabulario de la lengua general de todo el Perú llamada lengua qquichua o del Inca de Diego González Holguín (1608). Sin embargo no se trata de una simple copia de la obra de su ilustre predecesor. Sancho de Melgar ha realizado un esfuerzo notable de reorganización de los datos caóticos del vocabulario de González Holguín. Aquí como en otras partes del manuscrito, se puede observar que es el deseo de ser eficaz que predomina. Se ha reformulado el léxico de González Holguín para que pueda ser fácilmente consultado por el estudiante de la lengua general y también por el autor mismo que, sin duda, lo utilizaba

4 Este tratamiento especial dado a la bilabial se debe probablemente al deseo de evitar la confusión entre $p-h\left[p^{h}\right]$ y $p h[f]$. 
para realizar sus traducciones de los Evangelios. La grafía de las traducciones ya no es la de González Holguín, con todas sus inconsistencias, sino la misma que el autor propone en sus Reglas de la ortographia Indica (f44v-f45v). Hay también elementos originales, por ejemplo la entrada castellana coito ocupa una columna entera de su vocabulario mientras que ni aparece en el de González Holguín.

\section{LA TRADUCCIÓN DE LOS EVANGELIOS}

El extracto del Evangelio de Mateo que presento aquí fue escogido por su legibilidad. Sin embargo, no representa idealmente el estilo de Sancho de Melgar, Ileno de erudicón barroca. El inicio de otro texto Et Deus erat Verbum [f64r], que desafortunadamente en la fotocopia a mi disposición es en gran parte ilegible, dará una idea más precisa de la extraña mezcla del latín y del castellano, de citas de los evangelistas y de sus exegetas, que caracteriza su texto. El autor indica la traducción al quechua de las citas del Evangelio por un asterisco. Los pasajes en cursivas están subrayados en el original. Se discute del sentido preciso de cada elemento del texto latino antes de enfrentar su traducción para la cual frecuentemente se propone una o dos formas alternativas. Así camarccan (/ kamarqan/), empleado con el sentido de «creó» tiene como variante cachirccan (/kachirqan/) «hizo que existiera» y caccllapas (/kaqllapaŝ/) «todo lo que exite» alterna con kayñijoccllapas (/kayñiyuqllapâ̂/) «todo lo que tiene existencia». La justificación para sus traducciones está expuesta en detalle como también la propiedad gramatical de la estructura escogida. Las barras oblicuas separan los renglones del original. Pongo entre corchetes la transcripción normalizada de las traducciones al quechua. Los signos $<>$ encierran enmiendas del autor agregadas a su texto. Las palabras en negritas son tentativas de interpretar formas ilegibles de la fotocopia.

\section{Extracto de la Luçerna Yndyca del Dr Estevan Sancho de Melgar y Santa Cruz [f. 64r a f. 66v]}

«Tercera proposition: Et Deus erat Verbum.

Verborum constructio (dize Mald[onado]) ita ordinanda est, vt Verbum sit sub-/ iectum, Deus attributum; transpositis verbis: [1] Verbum erat Deus. ${ }^{*}$ Diospa churin Verbo ñisccari Diostaccmi cachcarccan. [Diospa churin Verbo ñiŝqari Diostaqmi kachkarqan]

V. 2. Hoc erat in principio apud Deum.

*Cay ñispa churin Verbo ñisccacca manaracc hinantin pacha teccsiscca / captin, Diospi cachcarccan [kay ñiŝpa churin Verbo ıniŝqaqa manaraq hinantin pacha tiqsiŝqa kaptin Diospi kachkarqan]; aut aliter vt supra in prima et secunda propositione.

V. 3. Omnia per ipsum facta sunt: et sine ipsa factum est nihil quod factum est. 
Praepositio per (dize Cornelio) cum dicitur per ipsum hîc non significat / causam instrumentalem, aut ministrum, quasi verbum fuerit, instrumentum, vel / minister Dei per quod creavit omnia. Y assi no se ha de traduzir con raycu, [ni] con / genitivo que corresponde a nuestra a .l. ab. Oygan a Cornelio: quaeres (dize) cur ergo / S. Ioannes potius dicat per Verbum omnia facta esse, quam à Verbo? Rs. Primo, / vt significet Verbum esse ideam rerum creatarum, iuxta quam Pater cum Filio cre-/avit omnia: secundo, proprie dicit per Verbum omnia esse facta; quia Verbum / â Patre accipit cum essentia divina omnipotentiam, et actionem eamdem nu-/mero, qua simul cum Patre omnia creat. Con cuyo fundamento se hara este pe-/rifrasi: In Juntamento con el crio el Padre todas las cosas: y sin el nada de / lo que tiene ser fue hecho. *Dios yayacca payvvan vvaquilla ima hayccactapas ca/marccan .I. cachirccan: mana payvvancca ima caccllapas .I. caynijoccllapas ma-/nam rurasccachu .l. camasccachu carccan. [Dios yayaqa paywan wakilla ima hayk'aktapaŝ kamarqan (o kachirqan); mana paywanqa ima kaqllapaŝ (o kayniyuqllapaŝ) manam ruraŝqachu (o kamaŝqachu karqan.] Y para comprobacion de que arriba de-/be dezirse cum ipso, atiende a Sylverio hîc num. 48. loannes id quod dixi-/rat, per affirmationem, repetit per negationem. La $\mathrm{n}<\mathrm{e}>$ gacion es sine ipso, preci-/samente sera cum ipso la afirmacion. Notando que ima caccllapas .I. cay nijocclla-/pas equivale gallardamente a quod factum est q. d. Nihil quod est, aut / habet esse sine ipso factum est».

\section{Referencias citadas}

AVENDAÑO, F. de, 1649 - Sermones de los misterios de nuestra santa fe católica en lengua castyellana y en la general del Inca; Lima.

ÁVILA, F. de, 1647-1648 - Tratado de los Evangelios que la iglesia propone en todo el año, 2 tomos; Lima.

GONZÁlEZ HOLGUíN, D., 1608 - Vocabulario de la lengua general de todo el Perú Ilamada lengua qquichua o del Inca, 707 pp.; Lima: Universidad Nacional Mayor de San Marcos.

MIDDENDORF, E.,1970 [1890] - Gramática Keshua, traducida del alemán por Ernesto More; Lima.

RIVET, P. \& CRÉQUI-MONTFORT, G., 1951 - Bibliographie des langues aymará et Kicua, 1540-1875, 499 pp.; Paris: Université de Paris, Institut d'ethnologie.

SANCHO DE MELGAR, E., 1691 - Arte de la lengua general del ynga llamada Qquechhua; Lima. 


\section{Anexo 1}

\section{Luçerna Yndyca [44v]}

\section{Reglas de ortographia Indica, para que el idiomista que aprende / por preceptos escriua con perfeccion y pronuncie con alguna pro/ priedad}

Num. 1. La buena pronunciacion (dize nuestro Nebrixa en su Arte) pende de la / buena orthographia: y siendo esta tan necessaria en la lengua Latina, cuya con-/ textura de sylabas concuerda con nuestra Cartilla Castellana, mucho mas lo serâ / en esta, cuyas dicciones apenas pueden deletrarse.

2 Faltan de nuestro Alphabeto a este idioma B. D. F. G. J. X pero tiene Cc. qq. / hh. tt. vv. th. p-h. cuyo vso es como se sigue.

3 De quatro maneras pronuncia el Indio la C. La primera sencilla como en Cas-/ tellano y Latin. vt cani. La segunda suave, como z. vt çaça, ȩøeç çocco. La tercera / con aspereza pronunciando desde las fauces, vt ñocca, ccam. La quarta tambien aspera, pe-/ro del paladar para fuera, vt ccatini. Algunos que han impresso han escri-/to esta vltima con K. ignoro el fundamento. Dos razones pudieran dar: o que el Indio / la auia escrito assi, o que la K. tiene la fuerça que le prohijan: la vna falta porque / el Indio no escriuio: a la segunda pregunto, si Kalendae y Kyrie tienen la aspereza que / ccatini? Lo cierto es que no: luego no se debe vsar. Solo me persuado que para variar / de pronunciacion la vsaron ad placitum. Yo digo que mas proprio es escriuir con $c c /$ y $q q$, y la propriedad la eñseñarâ el tiempo y la atencion a los natiuos, quando ha-/blan: porque es cierto que es imposible aprenderlo sine viuae vocis oraculo.

4 La q doblada tiene la pronunciacion como la c doblada de la quarta orden del / num. antecedente, vt qquevvini, ruqqui.

5 . Tiene otra prononciacion de .q. ante .h que se haze quasi separando aquella de / esta, vt vtq-hu el algodon.

6 En la $P$ ay tambien variedad: vnas vezes se pronuncia sencilla y $\sin$ fuerza, vt pipas, / otras hiriendo con fuerza los labios (y esta escriuiremos con pp) como ppacha, el vestido, / a distincion de pacha, el tiempo, de otras escriuiremos con ph pero no sonara quasi / $f$ como en propheta, sino que al parecer se pronuncia la $p$ separada de la $h$, co-/mo $p$-hucuni soplar, y para pronunciarla se hiere vn labio contra otro, aspirando / con ayre para la $h$.

7 Quando a la $p$ se sigue $r$ haze sonido de $F$ pero no liquesce la $R$ como en Cas- $/$ tellano y Latin, vt f...f... Africa, sino que la vocal antecedente se arrastra la / $\mathrm{R}$ y la $\mathrm{R}$ hiere suaue en la vocal subsequente, vt chap-rini, hap-ra, cuya pronuncia-/cion suena quasi chafrini, hafra. //

[45r]

8. Si la $R$ se hallare post $C$ (obseruando en la prononciacion la separacion de $P$ ante $R$ ) / tampoco hiere, y haze sonido quasi de G, vt chac-ra, roc-ro: y por esta 
precision de no herir / los sonidos $P$, y $C$ en la $R$. ningun vocablo de este idioma tiene primera sylaba que comien-/za con pra, pre, \& ni con cra, cre, por no auer vocal antecedente que arrastre $P$, o $C$.

9. Lo mismo se obseruarâ quando a la $C$ se siguen II, que sig vt chocc-llo, que se prononciarâ / quasi chog-llo: Y por esta causa he juzgado ser preciso separarlas con raya en medio, para que el / que no es natiuo sepa que es vna sola voz, pero que no ha de herir en los liquidos. Lo mismo se obser-/uarâ con la $Y$ que con raya antes no se junta con la consonante antecedente, y con raya despues, no / hiere en la vocal subsequente, vt tap-y-a, que se pronunciarâ q. d. tafia.

10. La c ante y diuidida con raya suena como G, vt vvac-y-ani. q. d. wagiani.

11. La tt doblada se pronuncia hiriendo con fuerza la lengua en los dientes, vt ttica la flor, / a distincion de tica el adobe: ttacani derramar cosas aridas, y tacani golpear.

12. He tenido suficiente motiuo para vsar de $v v$ doblada, como en vvavva, vveque, vvira, / huyendo de escriuir (como todos lo han hecho hasta aora) huahua, veqque, vira. Porque si / leemos el hua, hue, \&. como pronuncia el Castellano hueuo, huerta; mas suena $G$ que / otra letra, pues de ordinario dizen los Castellanos: gueuo, guerta, como a Huamancca, / y Huancavvillcca s Guamanga y Guancavelica. Si leemos va, ve, vi, \& parece $B$. heri-/do sin labios suena, como vara, verdad, vino, \&. Vno y otro dista mucho de lo que el / Indio pronuncia: luego no se deue escriuir como hasta aora. A que se llega que confi-/riendo este punto con vn sujeto muy docto, de singular ingenio, gran erudita, insigne no so-/lo en los idiomas Latino, Germanico, Frances, y otros desta Europa, sino tambien consuma-/do en los dos generales de este reyno qquechhua y Aymara, me asegurô que la pronun-/ciacion del Indio con estas sylabas era la misma que el vva, vve; vvi de los estrangeros.

13. La $H$. doblada post $C$ tiene distinta pronunciacion de la sencilla, vt michha, muchha.

14. Varios vocablos finalizan en ch como ach, ichach, achuch: pronuncionanse cuy-/dando de que no se expresse vocal despues de la $h$, sino que se finalize en esta sola.

15. No ay I sencilla en este idioma: y se ponen por objecion la interjecion alalay, que / todos han escrito con vna, respondo que no son sino dos allallay, que no soñarân como lla-/mar, llenar, et alia, sino como se estuviera escrito, al-lal-lay.

16. Tampoco tiene el Indio $R$ doblada: y assi, que este en principio o medio de / diccion suena lo mismo que nuestra $R$ en medio: por lo qual en rimarini, ruru, ram-/ram tienen la misma suauidad en las primeras, que en los medios, y no muy singular / pues en Italiano, y otros idiomas sucede lo mismo.

17. Ninguna voz de este idioma tiene pronunciacion aguda como en Castellano, entrô / con acento en la vltima, vt entrô, enseñê, amarâ. Todas las penultimas son largas como / < docebo $>/ /$

[45v]

docebo, labores, sin que aya penultima breue, como tempora, dominus, dabitis, sino es la vo-/cal ante vocal, vt onccoy, hamuy, ppunchau. 
18. Debese obseruar que ay muchas dicciones, que se componen te ad con dos, tres y mas / particulas como churijquicunactavvampas, donde se halla churi el hijo, iqui possessiuo. que / corresponde a tuus. cuna que lo pluraliza, cta que lo haze acusatiuo, y vvan con pas que son / conjunciones. Si esta o semejantes dicciones se huuiesen de diuidir en fin de renglon, se / partiran con raya, porque juntas gerunt vide vnius: y aunque de la diccion de arriba / churi, seorsum sumpta, significa filius, pero como las particulas que restan, vna le constitu-/ye possesso, otra plural, otra acusatiuo, y otras le conjuntan con diccion o clausula antece-/ dente, en fin de linea se le pondra a churi (o a qualquiera de las otras) raya para que se-/pa el que lee, no ha de parar alli, sino passar a la linea siguiente a buscar las sylabas restan-/tes, que le constituyen vn solo vocablo: a la manera que en Latin partimos en fin de linea / (quando se ofrece) a quam-obrem, sic-ut; quominus et alia quo-minus et alia.

19. Vltimamente note que el Indio confunde la $o<\operatorname{con}>$ la $v$, y la e con la $i$ : y assi, o ya en / impressiones, o ya oyendo al Indio notarân la variedad en vn mismo vocablo, porque v-/nos dizen oncconi y otros vnccuni: vnos reccini, y otros riccini. Lo cierto es que en el / Cuzco la pronuncian tan indiferente al sonido, que no se puede percebir si es vna, $v$ otra. / Esto ha mouido a los que han escrito Artes de este idioma a enseñar que quando la raiz del / verbo fuere $i$ como en puri-ni, con el participio de presente se mude en e, vt purecc: y / y [sic] si la raiz es $v$, se conuierta de $o$, vt en puñu-ni, puñocc. En este particular fijan al / vso del pais, o no le fijan, que tambien entenderan al que dize vvañucc, como al qu pro-/nunciare vvañocc. Y no es entre solos Indios esta confusion de letras, pues vemos en nues-/ tro Romano Castellano, que auiendo tenido su origen en la lengua Latina, de porta / dixeron puerta, de curta, corta, de timor temor: y aun en nuestro siglo oymos Sujetos / Castellanos, no Sayagueces, sino bien eruditos y rethoricos que dizen tiniendo y puniendo, / pro teniendo y poniendo.. Y en nuestro idioma vimos variar la raiz y de dezir sale / dijo, digo, \&. y de poner; salen puse, pusiera \&. y otros innumerables.

\section{Anexo 2}

Luçerna Yndyca, [182r <130>]

Dominica decima septima post Penticosten.

Evang. 108. Enseña lesus qual es el principal mandamiento de la ley: / confunde a los Phariseos con vna pregunta. Matt. 22.

\section{34. Accesserunt ad lesum Pharisaei:}

* Phariseocuna lesusman cayllaycurccan:

V. 35. Et interrogavit eum vnus ex eis legis doctor, tentans eum.

* hucñin camachicuscca simi yachayçapa payta vvateccaspari, tapurccan. // 
[182v]

V. 36. Magister, quod est mandatum magnum in lege?

* Yachachicamayocc, mayccanmi camachicuscca simipi collananñin camachicusccacca?

V. 37. Ait illi lesus: Diliges D(omi)num Deum tuum ex toto corde tuo, et in tota anima tua, et / in tota mente tua.

* lesus payman ñirccan: Apu Diosñijquiman soncco canqui tucuy sonccoyquivvan, tu-/cuy animayquivvan tucuy yuyayñijquivvampas.

V. 38. Hoc est maximum, et primum mandatum.

* Caymi collananñin camachicusccacca, ñaupaccñintaccmi.

V. 39. Secundum autem simile est huic: Diliges proximum tuum sicut te ipsum.

* Isccayñeqquen $* r i$ cayman $<m i>$ ricchaccmi<cca $>$ : Runamacijquicta .I. ccamhina runacta / quiquijquictahina munanqui.

V. 40. In his duobus mandatis vniversa lex pendet, et Prophetae.

* Tucuyñin camachicuscca simicca, Prophetocunap qquellccasccampas cay isccay cama-/chicuscca simipi vvisccacun.

V. 41. Congregatis autem Pharisaeis, interrogavit eos lesus,

* Phariseocuna ña huñunacuptin, lesus paycunacta tapuspa,

V. 42. dicens: quid vobis videtur de Christo cuius filius est? Dicunt ei: David.

* Nirccan: Messias Christomanta imañinquichicc? Pip churinmi? Payman ñirccan-/cu: Davidpa.

V. 43. Ait illis quomodo ergo David in spiritu vocat cum Dominum, dicens:

* Paycunaman ñirccan: Imahinatacc ari Davidcca Espiritu Santop çamaycusccan

I yachachisccan payta Apu, vvac-yaspa, ñirccan.

V. 44. Dixit Dominus Domino meo: Sede à dextris meis, donec ponam inimicos tuos scabe-/llum pedum tuorum?

* Apu Dioscca Apu Christosman ñirccan: Pañañeqquijpi tijaycuy, checneqqueyquicu-/nacta chaquijquip çaruchacunampacc churaycunaycama. Vt eos (dize Corn[elio]) quasi manci/pijs dominijs, imo eos calces quasi scabellum pedum tuorum.

V. 45. Si ergo David vocat eum Dominum, quomodo filius eius est?

* David ari payta, Apu, vvac-y-an chaycca, imahinam paypa churinmi?

V. 46. Et nemo poterat ei respondere verbum: Neque ausus fuit quisquam ex illa die / eum amplius interrogare. //

[183r]

ñiptinsi manam huc simillactapas cutipanancu yachacupurccanchu: manatacc pipas / chay ppunchaumantapacha payta astavvan * tapuyta checcancharccurccanchu. 
Texto quechua normalizado

Fariseokuna Jesúsman qayllaykurqan. Hukñin kamachikuŝqa ŝimi yachaysapa payta watiqaŝpari tapurqan: «Yachachikamayuq, mayqanmi kamachikuŝaa simipi qullananñin kamachikuŝqaqa?» Jesús payman ñirqan: «Apu Diosñiykiman ŝunqu kanki tukuy ŝunquykiwan, tukuy ánimaykiwan tukuy yuyayñiykiwanpaŝ. Kaymi qullananñin kamachikuŝqaqa, ñawpaqñintaqmi. Iŝkayñiqinri kaymanmi rikch'aqqa5: Runamasiykikta (o qamhina runakta) kikiykiktahina munanki. Tukuyñin kamachikuŝqa ŝimiqa Profetakunap qillqaŝqanpâ̂ kay iŝkay kamachikuŝqqa ŝimipi wi[ch]q'akun6». Fariseokuna ña huñunakuptin Jesús paykunakta tapuŝpa ñirqan: «Mesías Cristomanta imañinkichik? Pip churinmi?» Payman ñirqanku: «Davidpa». Paykunaman ñirqan: «lmahinataq-ari Davidqa Espíritu Santop samaykuŝaan yachachiŝqan payta 'Apu' waqyaŝpa ñirqan? Apu Diosqa apu Cristosman ñirqan: 'Pañañiqiypi tiyaykuy, chiqniqiykikunakta chakiykip saruchakunanpaq churaykunaykama. David-ari payta waqyan chayqa, imahinam paypa churin \{mi\}?» Ñiptinŝi manam huk ŝimillaktapaŝ kutipananku yachakupurqanchu. Manataq pipaŝ chay p'unchawmantapacha payta aŝtawan tapuyta chiqancharqurqanchu.

5 La distinción en el empleo de -mi y de -qa es una de las preocupaciones gramaticales principales de Sancho de Melgar. Su corrección de este pasaje refleja sus vacilaciones.

6 La grafía empleada por Sancho de Melgar vvisccacun sugiere una evolución de la africada /ch/ semejante a la pronunciación del cuzqueño actual: wisq'akun. Sin embargo, en su léxico se encuentra la forma más ortodoxa: «Cerrar Vvichccani».

7 Según sus propios criterios, el autor habría tenido que suprimir el segundo -mi. 